\title{
Nosema ceranae infection enhances Bifidobacterium spp. abundances in the honey bee hindgut
}

\author{
Yakun Zhang, Xiaoxiao Lu, Shaokang Huang, Lina Zhang, Songkun Su, \\ Wei-Fone HuAnG
}

College of Bee Science, Fujian Agriculture and Forestry University, Fuzhou 350002 Fujian, China

Received 5 July 2018 - Revised 15 January 2019 - Accepted 19 March 2019

\begin{abstract}
Nosema ceranae has become the dominant Nosema pathogen in the honey bees, Apis mellifera. It primarily infects and reproduces in midguts and reproduced $N$. ceranae spores travel to hindguts and leave hosts with feces. Hindguts have structural support for bacterial symbionts and biofilms, which harbor the densest bacteria of the entire bee. Although some bacteria were inhibitory to $N$. ceranae in the laboratory trials, $N$. ceranae disease is not apparently hurdled by hindgut bacteria. We suspected $N$. ceranae infection may affect hindgut bacteria populations to favor the spore survival. In this study, hindgut bacterial microbiomes of defecated foragers were analyzed using a pyrosequencing method. The results suggested Bifidobacterium spp. abundances were significantly and positively associated with $N$. ceranae infection. To confirm the association in a larger sample size, we used caged bees and qPCRs that quantify bifidobacteria and lactobacteria. The same association was found in emptied hindgut samples, but not significant in feces samples. Bifidobacteria in feces might increase when bifidobacteria on hindguts are significantly enhanced by $N$. ceranae infection. Although we have not identified the specific causes and mechanisms of this association, the results suggested that indigenous Bifidobacterium spp. in honey bee hindguts may have no prevention effects on $N$. ceranae disease.
\end{abstract}

\section{bacterial microbiome / probiotics / Nosema / actinobacteria}

\section{INTRODUCTION}

Nosema disease, nosemosis, is the most common gut disease in adult honey bees. Nosema is a genus of Microsporidia, which often causes problems in mass rearing insects, including laboratory insects, silkworms (Becnel and Andreadis 1999), and honey bees (Chen and Huang 2010; Fries 2010). The disease is usually chronic without a visible sign or symptom until the late stages. Two Nosema pathogens, Nosema apis and $N$. ceranae, have been identified in honey bees. $N$. apis was first found in Apis mellifera in the early twentieth century (Zander 1909), and it was

Corresponding author: W. Huang, wfhuang@fafu.edu.cn

Manuscript editor: Yves Le Conte considered the only Nosema pathogen in honey bees before the discovery of N. ceranae in A. cerana (Fries et al. 1996). $N$. ceranae can infect both A. mellifera and A. cerana (Fries 1997), but the first noted infection in A. mellifera was late in 2005 (Huang et al. 2007). $N$. ceranae was then quickly identified in different honey bee populations all over the world (Huang et al. 2008; Klee et al. 2007). It has become the dominant or the only Nosema pathogen in most A. mellifera populations (Fries 2010), and the earliest $N$. ceranae record in A. mellifera has been traced back decades before the species description (Teixeira et al. 2013).

Nosema disease in honey bees was considered to occur only in adult guts and to transmit majorly horizontally (Bailey and Ball 1991; Huang and Solter 2013). The disease has been controlled by fumagillin for decades, but fumagillin is toxic to bees and less efficient on $N$. ceranae than $N$. apis 
(Huang et al. 2013). Gut microbiomes could be a new solution for Nosema disease. Some studies have shown certain bacteria have antagonistic or inhibiting effects on $N$. ceranae. Foregut lactobacteria (Vásquez et al. 2012) and bacterial organic acids (Maggi et al. 2013) reduce $N$. ceranae infection intensity. Feeding exogenous probiotic bacteria mixed with Lactobacterium and Bifidobacterium spp. decrease $N$. ceranae infection intensity as well (Baffoni et al. 2016).

Honey bee hindguts harbor the highest density of bacteria (Engel et al. 2012). It is a cuticular organ and has structural supports to harbor microbes and biofilms. Hindgut microbiomes are largely inhabited by Lactobacterium and Bifidobacterium spp., same species found in other parts of the alimentary canal (Engel et al. 2012). Feces that are fermented by hindgut microbiomes were the most common media for $N$. ceranae transmission (Huang and Solter 2013). Although some hindgut bacteria showed inhibiting effects to $N$. ceranae in the laboratory, $N$. ceranae spreading seemed not be affected. $N$. ceranae has become the dominant pathogen for decades (Chen and Huang 2010; Fries 2010; Paxton 2010; Teixeira et al. 2013); thus, $N$. ceranae might have changed or co-evolved with hindgut microbiomes.

Two stages of investigations of hindgut bacterial microbiota were carried out in this study. Initially, we collected naturally $N$. ceranae infected and uninfected forager bees to investigate the differences of microbiome within hindguts. Bacterial microbiota of the hindguts was compared using a pyrosequencing method. qPCRs and samples from caged bees that were reared and inoculated in laboratory settings were used to confirm the pyrosequencing results in a larger sample size. We aimed to investigate any specific association between $N$. ceranae infection and hindgut bacterial microbiota.

\section{MATERIALS AND METHODS}

\subsection{Sample collection and DNA isolation}

The honey bees used in this study were common subspecies in Fujian, China, with most phenotype characteristics of Apis mellifera ligustica and occasionally reproducing dark color bees. We selected three colonies of different origins from the apiary at Fujian Agriculture and Forestry University. Foragers with combed pollen were collected in front of the hive entrances. The collected foragers were kept on ice before dissection; bees that had lost pollen on corbicula hair were discarded. We collected approximately $30-50$ foragers for each colony.

We previously only found $N$. ceranae in our apiaries using the PCR screenings described in a previous work (Huang and Solter 2013) and no $N$. apis detection in this part of China (Liu et al. 2008); therefore, Nosema species identification was not done for each bee, but the infection intensity was evaluated for each bee. The midgut of each forager was pulled out and ground in one Eppendorf tube (Huang and Solter 2013). Homogenized midgut samples were centrifuged at $6000 \times g$. Samples were visually screened by the spore pellets in the bottom; we selected samples with no visible spore pellet and those with large spore pellets for the further exam. Infection levels were then evaluated by spore counting under a phase-contrast microscope using a hemocytometer (Bailey and Ball 1991). Samples with no spore were grouped as no apparent infection samples, and samples with more than 10 million spores were grouped as heavily infected samples as infection intensity may have reached the plateau phase, > $10^{7}$ spores per bee (Forsgren and Fries 2010; Huang and Solter 2013). Four to six foragers of both the infected or uninfected group were identified from each colony, and the hindguts were used for DNA extraction and pyrosequencing. Six samples in total were used in the subsequent pyrosequencing. DNA of the pooled hindgut samples was extracted using traditional DNA extraction methods: proteinase $\mathrm{K}$ treatment followed by phenol/chloroform and alcohol precipitation. The precipitated DNA pellets were washed twice using $70 \%$ alcohol and dried in a laminar flow hood. After fully dissolving the DNA in nuclease-free water, the DNA quantity and quality were measured using Nanodrop (Thermo Fisher).

\subsection{Pyrosequencing for bacterial microbiome}

The pyrosequencing method was applied to sequence all PCR amplified bacterial 16S rRNA 
V3 region. PCR used a two-step method (95C 20S, followed by 55C 30S; 40 cycles) using Taq premix (TAKARA). One hundred nanograms of isolated DNA was used in each PCR $(50 \mu \mathrm{l})$. Primer set, 341F-B27 (ATTCCTACGG GAGGCAGCAG) and 518R-B25 (ACTGAT TACCGCGGCTGCTGG) were used; the underlined sequence is the barcodes used to share sequencing capacity in Illumina Hiseq. Sequence reading was done on the Illumina Hiseq 2500 platform, and preliminary data analyses were assisted by Novogene, Beijing. The datasets were compared with bacterial databases, and only the sequences matching bacterial $16 \mathrm{~S} \mathrm{~V} 3$ region were included in the following analyses.

We used $97 \%$ identity as the threshold for the sequences from the same species. Species relative abundance was annotated for each sample. To visually reveal the similarity among the samples, we grouped the samples by species at phylum level using UPGMA (unweighted pair-group method with arithmetic mean) analyses with weighted unifrac and unweighted unifrac methods, with the weighted unifrac method having bacteria abundance as a factor during grouping. $T$ test and linear discriminative analysis effect size (LEfSe) were applied to find the significant differences between the bacterial microbiomes of $N$. ceranae heavily infected and uninfected bees.

\subsection{Laboratory cage rearing and $N$. ceranae inoculation}

We noted the pyrosequencing results did not agree with published reports ( $\mathrm{Li}$ et al. 2017) that suggested no significant difference found in $N$. ceranae infected bees. To confirm the association between $N$. ceranae and bifidobacteria and investigate the differences in the experiment designs, similar trials of caged bees that used in Li et al. (2017) were conducted. In addition, we used qPCRs ( $\mathrm{Li}$ et al. 2012) in a larger sample size (we obtained approximately 40) to confirm the association with less cost than that of the pyrosequencing method. Furthermore, the forager samples were those returning to hive with empty hindguts, defecated during foraging flights, whereas caged bees have full hindguts, no defecation in cages. Therefore, we separated the hindgut linings and feces of caged bee samples to investigate which part harbors the significantly increased bifidobacteria populations.

The cage rearing method was identical to that used in Huang and Solter (2013) with locally purchased materials in Fuzhou. Bees were harvested from capped frames, kept in a growth chamber, entirely dark at $34{ }^{\circ} \mathrm{C}$ and $70 \%$ relative humidity. The capped frames were pull out from two colonies (not the same colonies used for pyrosequencing), in different seasons, May and October. The harvested newly emerged bees were held in the cages and transferred into a $30{ }^{\circ} \mathrm{C}$ growth chamber. Five-day-old bees were anesthetized on ice and separated into 200 bees per cage. A group inoculation method (Huang et al. 2015) was used; approximately $10^{5}$ spores were fed to each bee in $24 \mathrm{~h}$. At 16 and 20 dpi, 20 alive of either inoculated or control bees were harvested and dissected. Dissected hindgut linings were gently washed twice in PBS after collecting feces samples. Samples that were failed to obtain clear separation of hindgut lining and feces were discarded. Hindgut and feces DNA were then extracted by the CTAB/proteinase $\mathrm{K}$ treatment followed by phenol/chloroform method, as described in previous sections. CTAB was added for digested plant materials in the feces.

\subsection{Quantification PCR for bifidobacteria and lactobacteria abundances}

Quantification PCR of bifidobacteria and lactobacteria ( $\mathrm{Li}$ et al. 2012) was employed. We applied the same amount of DNA to each qPCR (5 ng in a 5- $\mu$ l volume) and compared the quantification cycle, the cycle in which fluorescence signal exceeds the set threshold is termed quantitation cycle, $\mathrm{Cq}$ for short. We have applied the same forager bee DNA samples that used in pyrosequencing to the qPCR, and the results of bifidobacteria quantities were coincident. It suggested the qPCR and pyrosequencing results should be comparable.

The SYBR qPCR method and the primers, Bifido F/R and Lacto F/R (Li et al. 2012), were used. We used SYBR green premix (ChamQ, Vazyme) and CFX384 (Bio-rad) and adjusted the conditions by the results of gradient temperature 
tests on CFX384. To use both Bifido F/R and Lacto $F / R$ primer sets on the same plate and have similar PCR efficiencies, the primer concentrations were also adjusted: $200 \mathrm{pM}$ for Bifido F/R and $450 \mathrm{pM}$ for Lacto $\mathrm{F} / \mathrm{R}$. Both $\mathrm{qPCR}$ reactions have efficiencies within the range of $90-100 \%$ using the two-step method (95C 10S, followed by $57 \mathrm{C} 30 \mathrm{~S} ; 40$ cycles).

We found the $\mathrm{Cq}$ values of either bifidobacteria or lactobacteria were significantly lower in feces samples. To compare the quantification results between hindgut linings and feces, we compared the results using relative quantification method $(\triangle \mathrm{Cq})$ that utilized lactobacteria $\mathrm{Cq}$ readings as internal references. The $\Delta \mathrm{Cq}$ results indicated the ratio between lactobacteria and bifidobacteria and transformed the results of feces and hindgut linings into comparable values.

The differences between the results were compared using one-way ANOVA, using IBM SPSS22 for the calculation.

\section{RESULTS}

Pyrosequencing resulted in 51,145 taxon tags on average, with 47,371-56,862 tags in the samples after removing poor quality sequence reads (primer sequence reads and read lengths less than $30 \mathrm{bp}$ ) and those not belonging to the bacterial 16S rRNA V3 region. Unique sequence readings (no other similar tag in the results) were excluded in the following analyses because bacteria should grow in populations. The remaining taxon tags were further grouped into 53-61 bacterial species in each sample according to $97 \%$ identity threshold. The UPGMA analyses for grouping the samples (Figure 1a, b) were similar between $N$. ceranae heavily infected and uninfected samples. Samples from the same colony had similar species of bacteria (Figure 1a) and the abundances across the phyla (Figure 1b). We used $T$ test to compare the abundances of different orders between infected and uninfected samples, and the abundance of Actinobacteria (the phylum of bifidobacteria) was the only phylum that showed a significant difference in abundances $(P=$ $0.008)$. Actinobacteria increased in the samples from heavily infected bees, and the rest orders had no significant change. Linear discriminative analysis effect size (LEfSe) also suggested bifidobacteria was the major group affected by the infection (Figure 2a, b). Although LEfse indicated Alphaproteobacteria (the order of lactobacteria) may be altered, this was not supported in the lower taxonomic ranks (Figure 2b).

We found both lactobacteria and bifidobacteria qPCR results were significantly lower in hindgut lining samples of infected caged bees, but not the feces samples (Figure 3a). Thirty-eight feces (13 from the trial in May and rest from the trial in October) and 40 hindgut (15 from May and 25 from October) samples of caged bees were included in the qPCR after discarding samples of poor DNA quality (OD $260 / 280<1.7$ ) or concentration $(<5 \mathrm{ng} / \mu \mathrm{l})$. Feces samples showed significantly lower $\mathrm{Cq}$ values of either bifidobacteria or lactobacteria comparing to that of hindgut lining samples (both $P$ values were less than 0.001 ), suggesting more bacteria existed in feces samples. Lower mean bifidobacterial $\mathrm{Cq}$ values were consistently found in infected bees (both hindgut lining and feces), but only hindgut lining samples showed significance in one-way ANOVA test $(P<0.001) . \Delta \mathrm{Cq}$ values indicated the ratio between lactobacteria and bifidobacteria in the samples (Figure 3b). Unlike the significantly lower Cq values in feces samples, we found the $\Delta \mathrm{Cq}$ values were similar between feces and hindgut lining samples $(P=0.664)$; however, feces samples were still insignificantly different between infected and uninfected $(P=0.142) . \Delta \mathrm{Cq}$ values in hindgut lining samples were still significantly different $(P=0.002)$ between infected and uninfected samples. The difference between infected and uninfected samples $(\Delta \Delta \mathrm{Cq})$ was 1.44 and 1.73 for the feces and hindgut samples, respectively.

\section{DISCUSSION}

The pyrosequencing and the qPCR results all indicated that $N$. ceranae infection significantly increases Bifidobacterium spp. abundances. The pyrosequencing results suggested approximately doubled Bifidobacterium spp. abundances in the emptied, naturally defecated, hindguts of heavily infected foragers. The qPCRs of caged bees also showed the same trend, especially in $\Delta$ Cq results. Pyrosequencing did not suggest a difference in 


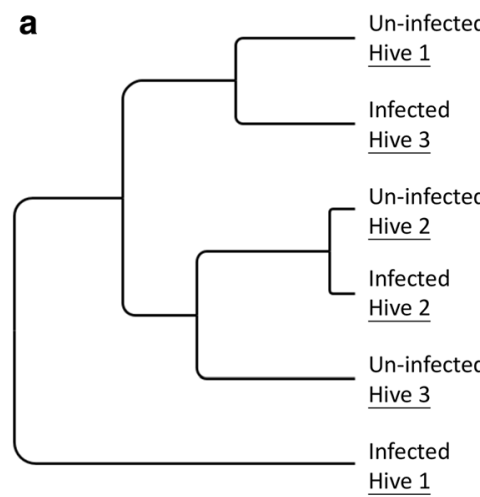

Unweighted Unifrac Distance $\begin{array}{lllll} & 0.125 \\ 0 & 0.025 & 0.05 & 0.075 & 0.1 \\ 0\end{array}$ b

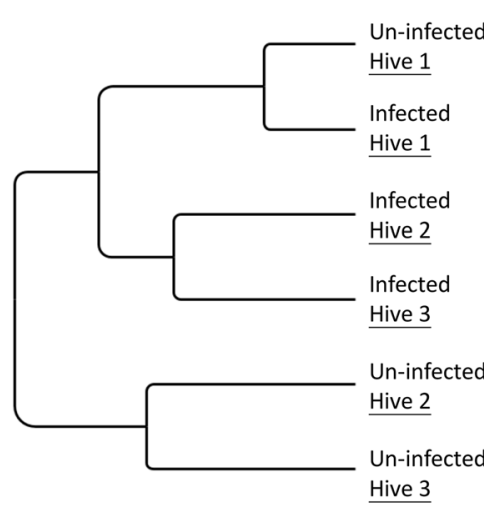

Weighted Unifrac Distance

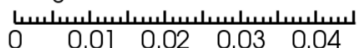

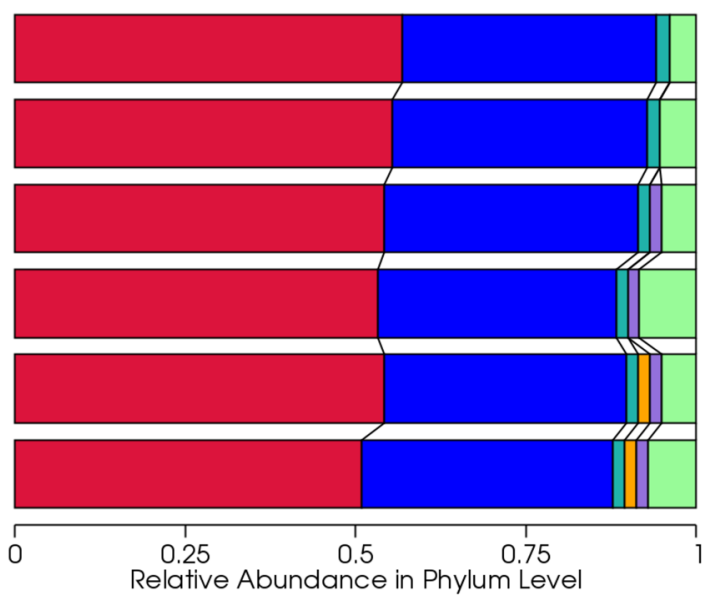

Proteobacteria Firmicutes Actinobacteria Cyanobacteria $\square$ Bacteroidetes Others

Figure 1. Unweighted pair group method with arithmetic mean (UPGMA) analyses to group the pyrosequencing results in unweighted unifrac distance (a) and weighted unifrac distance (b). UPGMA with unweighted unifrac distance (a) did not support any difference caused by $N$. ceranae infection, which suggested no specific association with the infection. Weighted unifrac distance (b ) analysis that adding abundance factor in the analysis separated hive 2 and 3 samples by the infection but not hive 1 .

lactobateria, but we found a significant increase of lactobacteria in infected caged bees using qPCR. We guessed the different primers that used in pyrosequencing and qPCRs could contribute to this difference; therefore, we compared the $\Delta \mathrm{Cq}$ results that leveled results using lactobacteria $\mathrm{Cq}$ values, and similar results, an approximately 3.3fold increase, were shown.

Field-collected foragers and caged bees may have different microbiomes in hindguts (Powell et al. 2014). Forager hindgut microbiomes developed naturally within hives and ingested food from natural environments, but caged bees have less than $24 \mathrm{~h}$ to obtain microbes from the brood frame and no contact with older bees and natural food sources. Even at the same age, microbiomes of caged bees may be less complicated than that of foragers; thus, caged bees could be more susceptible for the effects caused by $N$. ceranae infection. The results of qPCR did support this assumption that bifidobacteria increase more in infected caged bees than that of foragers.

Feces samples from caged bees have provided more results for identifying where the increased bifidobacteria located. We found no statistical difference between the feces samples of infected and 
a $\square$ Infected $\square$ Un-infected

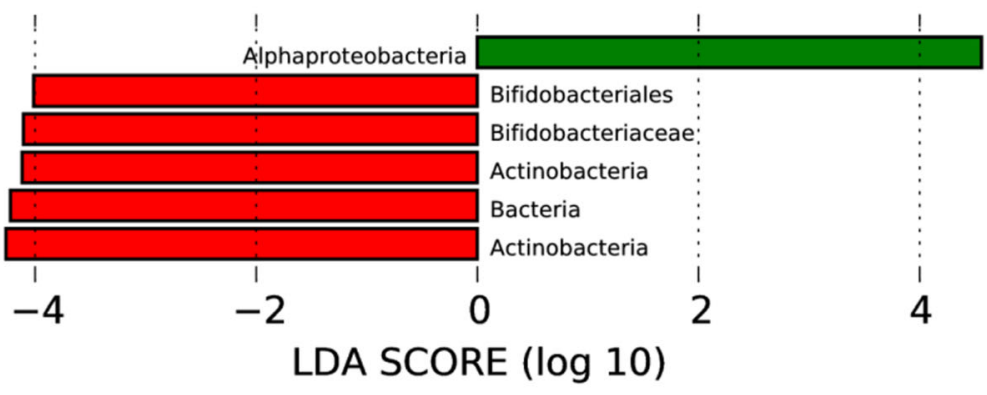

b

\section{Cladogram}

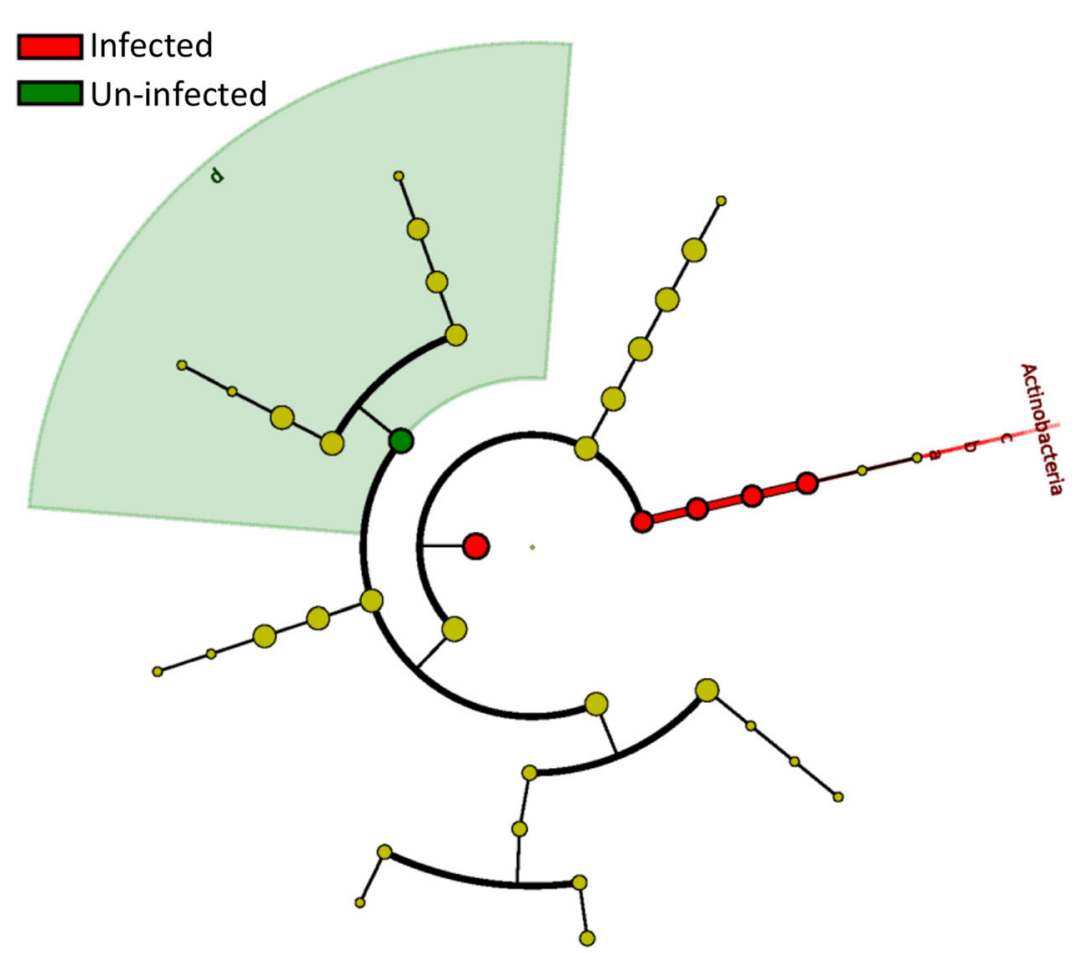

Figure 2. Linear discriminant analysis (LDA) effect size (LEfSe) analysis of the pyrosequencing results, LDA scores (a), and cladogram of the clusters (b). LDA scores (a) suggested all taxonomy classes of Actinobacteria were affected by $N$. ceranae infection, whereas Alphaproteobacteria was the only taxonomy class affected. The cladogram (b ) shows the single lineage of Actinobacteria in the pyrosequencing results, but Alphaproteobacteria has more diversity in the lower taxonomic ranks, which does not support the association with $N$. ceranae infection.

uninfected caged bees. Hindgut lining samples, on the other hand, had significantly higher bifidobacteria and lactobacteria quantities in the infected bees. These results suggested the bifidobacteria population on hindgut linings is the primary part affected by $N$. ceranae infection, not the feces. Bifidobacteria and lactobacteria populations on hindgut linings were directly affected by 
a

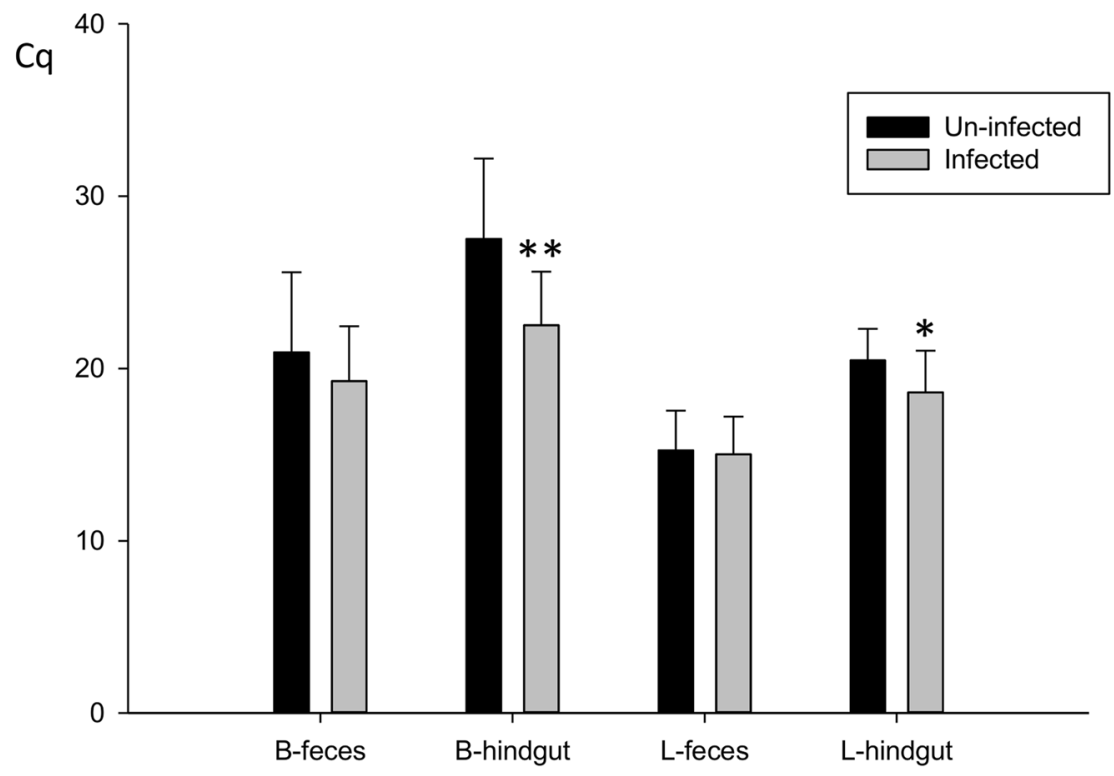

b

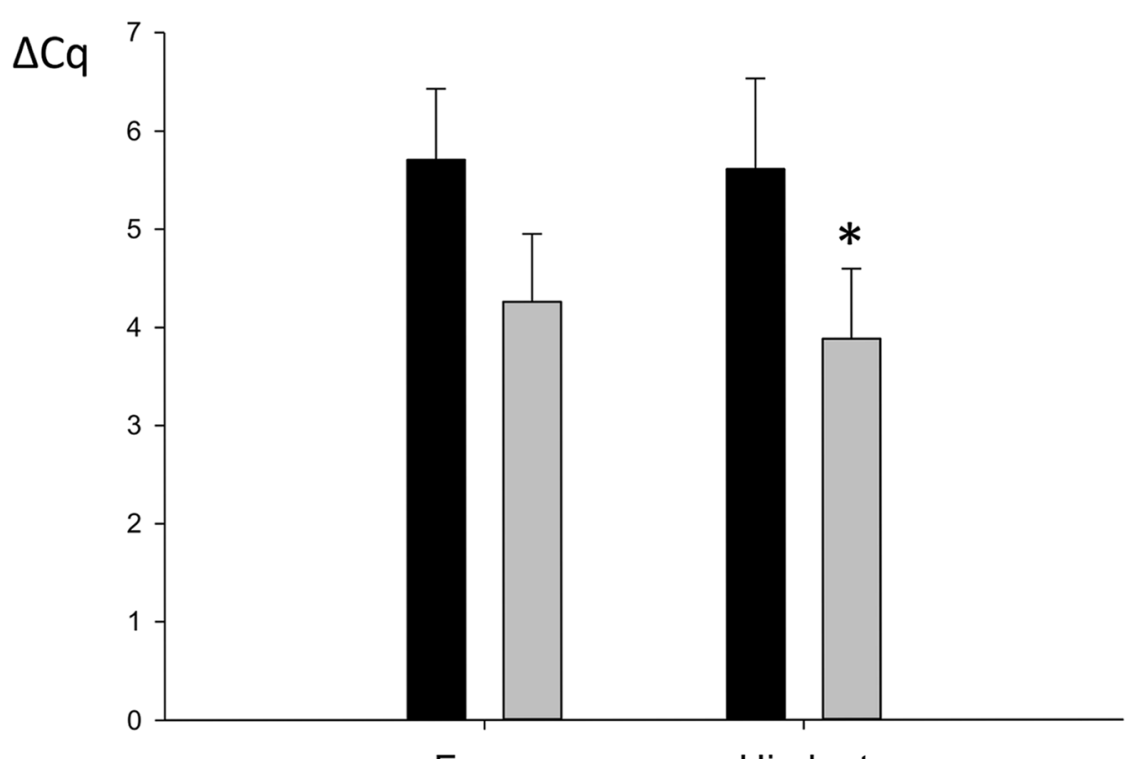

Feces

\section{Hindgut}

Figure 3. Quantification PCR results and Cq values of bifidobacteria and lactobacteria (a) and $\Delta$ Cq values (b); the lower values indicate the higher contents in the DNA samples. a B-feces and B-hindgut indicate the qPCR results of bifidobacteria in feces and hindgut lining samples; L-feces and L-hindgut indicate lactobacteria results. Both bifidobacteria and lactobacteria have significantly lower Cq, higher DNA copies, in hindgut samples, and the significance of Bifidobacteria results is quite robust $(P<0.001)$. b $\Delta \mathrm{Cq}$ values of feces and hindgut samples that indicate the ratio between bifidobacteria and lactobacteria. The results suggested both feces and hindgut samples have bifidobacteria population increase more than that of lactobacteria in infected samples. Infected hindgut samples have a significantly higher ratio of increase $(P=0.006)$. 
$N$. ceranae infection, and these populations might further change the bacteria composition in feces. Feces samples are complicated in composition, undigested food and debris. We speculated that the composition of feces might also affect the microbiomes, which may be the reason for the smaller and insignificant differences in qPCR results. The same reason may have contributed to the differences among our results and previous studies analyzing the microbiomes with feces ( $\mathrm{Li}$ et al. 2012; Li et al. 2017).

We have noted that the difference could be subtle between $N$. ceranae infected and uninfected bees because previous reports ( $\mathrm{Li}$ et al. 2012; $\mathrm{Li}$ et al. 2017) did not find any difference. Therefore, we selected the foragers with the spore numbers closer to fully developed infections (Forsgren and Fries 2010; Huang and Solter 2013) and used caged bees in the following trials to enhance any possible difference within hindgut microbiomes. Pyrosequencing results of forager hindguts provided a comprehensive bacteria microbiome comparisons between $N$. ceranae infected and uninfected bees, and the results suggested $N$. ceranae infection affects bifidobacteria abundance positively in hindguts. qPCR confirmed this association and suggested the significant alteration occurs only on hindgut linings, including ileum in this study, which provides structural support for bacterial symbionts and biofilms.

$N$. ceranae infection can occupy a large part of the midgut (Huang and Solter 2013) and affect the physiology and immune responses (Antúnez et al. 2009). Deficiencies of the infected midgut physiology may affect the composition of the undigested materials flowing into the hindgut, and it may further change microbiota compositions in hindguts. The normal bee gut microbiota has been associated with lower levels of infection with pathogens, which may indicate a beneficial role of the microbiota for the host bee (Kwong and Moran 2016). Lactobacterium and Bifidobacterium spp. are generally believed as probiotics in various animals and has been used as probiotics in A. mellifera as well (Evans and Lopez 2004; Forsgren et al. 2010). The occurrence of bifidobacteria and lactobacteria was positively correlated, which may reflect their similar substrate requirements or, possibly, a mutualistic relation (D'Alvise et al. 2017). However, exogenous
Bifidobacterium spp. were less effective in reducing $N$. ceranae infection comparing to exogenous Lactobacterium spp. (Baffoni et al. 2016). Our results suggested indigenous Bifidobacterium spp. in honey bee hindguts are not able to prevent $N$. ceranae spreading. The relationship between $N$. ceranae infections in midguts and bifidobacteria in hindguts is unclear. The increased bifidobacteria could maintain the homeostasis of the gut, which might also be the reason that fewer bees with diarrhea symptoms were noted in $N$. ceranae infections than in N. apis infections (Fries 2010). If the increased bifidobacteria population enhances the impacts and stimulations that directly linked to bee health and physiology (Kešnerová et al. 2017), it may increase host longevity. It is also beneficial to $N$. ceranae as well because of more time for its reproductions and transmissions. The association between bifidobacteria and $N$. ceranae may contribute to $N$. ceranae as being a widely spread and the dominant pathogen.

\section{AUTHORS' CONTRIBUTIONS}

WFH conceived the research and designed experiments; YZ and XL performed the pyrosequencing experiment; $\mathrm{YZ}, \mathrm{SH}$, and $\mathrm{LZ}$ conducted the qPCRs experiment; YZ, SS, WFH participated interpretation.

\section{FUNDING INFORMATION}

This study was funded by the National Natural Science Foundation of China (General Program: 31772681) and the Modern Agro-industry Technology Research System (No. CARS-45-KXJ3) of China.

L'infection à Nosema ceranae augmente l'abondance de Bifidobacterium spp. dans l'intestin grêle de l'abeille

Microbiome bactérien / probiotiques / Nosema / Actinobactéries

Nosema ceranae-Infektionen erhöhen die Abundanz von Bifidobacterium spp. im Enddarm der Honigbiene.

Bakterien-Mikrobiom / Probiotics / Nosema / Actinobacteria 


\section{REFERENCES}

Antúnez K., R. Martín-Hernández, L. Prieto, A. Meana, P. Zunino, et al. (2009) Immune suppression in the honey bee (Apis mellifera) following infection by Nosema ceranae (Microsporidia). Environ Microbiol 11 (9): 2284-2290.

Baffoni L., F. Gaggia, D. Alberoni, R. Cabbri, A. Nanetti, et al. (2016) Effect of dietary supplementation of Bifidobacterium and Lactobacillus strains in Apis mellifera L. against Nosema ceranae. Beneficial microbes 7 (1): 45-51.

Bailey L., B.V. Ball. (1991) 6-MICROSPORA AND PROTOZOA, Honey Bee Pathology (Second Edition), Academic Press, London, pp. 64-77.

Becnel J.J., T.G. Andreadis. (1999) Microsporidia in Insects, The Microsporidia and Microsporidiosis, American Society of Microbiology.

Chen Y.P., Z.Y. Huang. (2010) Nosema ceranae, a newly identified pathogen of Apis mellifera in the USA and Asia. Apidologie 41 (3): 364-374.

D’Alvise P., F. Böhme, M.C. Codrea, A. Seitz, S. Nahnsen, M. Binzer, et al. (2017) The impact of winter feed type on intestinal microbiota and parasites in honey bees. Apidologie 49: 252-264.

Engel P., V.G. Martinson, N.A. Moran. (2012) Functional diversity within the simple gut microbiota of the honey bee. Proceedings of the National Academy of Sciences 109 (27): 11002-11007.

Evans J.D., D.L. Lopez. (2004) Bacterial Probiotics Induce an Immune Response in the Honey Bee (Hymenoptera: Apidae). Journal of Economic Entomology $97(3):$ 752-756.

Forsgren E., I. Fries. (2010) Comparative virulence of Nosema ceranae and Nosema apis in individual European honey bees. Veterinary parasitology $\mathbf{1 7 0}(3)$ : 212-217.

Forsgren E., T.C. Olofsson, A. Vásquez, I. Fries. (2010) Novel lactic acid bacteria inhibiting Paenibacillus larvae in honey bee larvae. Apidologie 41 (1): 99-108.

Fries I. (1997) Protozoa, in: Morse R.A. (Ed.), Honey bee pests, predators, and diseases, 3rd ed., A.I Root Company, Medina, Ohio, USA, pp. 57-76.

Fries I. (2010) Nosema ceranae in European honey bees (Apis mellifera). Journal of invertebrate pathology 103 Suppl 1 : S73-79.

Fries I. F.F., Da Silva A., Slemenda S.B., Pieniazek N.J. (1996) Nosema ceranae n. sp. (Microspora, Nosematidae), morphological and molecular characterization of a Microsporidian parasite of the Asian honey bee Apis cerana (Hymenoptera, Apidae). Eur. J. Protistol. 32 : 356-365.

Huang W.F., L.F. Solter. (2013) Comparative development and tissue tropism of Nosema apis and Nosema ceranae. Journal of invertebrate pathology $113(1)$ : 35-41.

Huang W.F., J.H. Jiang, Y.W. Chen, C.H. Wang. (2007) A Nosema ceranae isolate from the honeybee Apis mellifera. Apidologie 38 (1): 30-37.

Huang W.F., M. Bocquet, K.C. Lee, I.H. Sung, J.H. Jiang, et al. (2008) The comparison of rDNA spacer regions of Nosema ceranae isolates from different hosts and locations. Journal of invertebrate pathology 97 (1): 9-13.

Huang W.-F., L.F. Solter, P.M. Yau, B.S. Imai. (2013) Nosema ceranae Escapes Fumagillin Control in Honey Bees. PLOS Pathogens 9 (3): e1003185.

Huang W.-F., L. Solter, K. Aronstein, Z. Huang. (2015) Infectivity and virulence of Nosema ceranae and Nosema apis in commercially available North American honey bees. Journal of invertebrate pathology 124 : 107-113.

Kešnerová L., R.A.T. Mars, K.M. Ellegaard, M. Troilo, U. Sauer, P. Engel (2017) Disentangling metabolic functions of bacteria in the honey bee gut. PLoS Biology $15: 1-28$.

Klee J., A.M. Besana, E. Genersch, S. Gisder, A. Nanetti, et al. (2007) Widespread dispersal of the microsporidian Nosema ceranae, an emergent pathogen of the western honey bee, Apis mellifera. Journal of invertebrate pathology 96(1): 1-10.

Kwong W.K., N. A. Moran (2016) Gut microbial communities of social bees. Nature Reviews Microbiology $14: 374-384$.

Li J., H. Qin, J. Wu, B.M. Sadd, X. Wang, et al. (2012) The Prevalence of Parasites and Pathogens in Asian Honeybees Apis cerana in China. PloS one 7 (11): e47955.

Li J.H., J.D. Evans, W.F. Li, Y.Z. Zhao, G. DeGrandiHoffman, et al. (2017) New evidence showing that the destruction of gut bacteria by antibiotic treatment could increase the honey bee's vulnerability to Nosema infection. PloS one 12 (11): e0187505.

Liu F., Q. Wang, P.-L. Dai, Y.-Y. Wu, H.-L. Song, et al. (2008) Natural stripe of microsporidia of honeybee in China. Chinese Bulletin of entomology 45 (6): 963966.

Maggi M., P. Negri, S. Plischuk, N. Szawarski, F. De Piano, et al. (2013) Effects of the organic acids produced by a lactic acid bacterium in Apis mellifera colony development, Nosema ceranae control and fumagillin efficiency. Veterinary Microbiology 167 (3): 474-483. 
Paxton R.J. (2010) Does infection by Nosema ceranae cause "Colony Collapse Disorder" in honey bees (Apis mellifera)? J Apicult Res 49(1): 80-84.

Powell J.E., V.G. Martinson, K. Urban-Mead, N.A. Moran. (2014) Routes of Acquisition of the Gut Microbiota of the Honey Bee Apis mellifera. Appl Environ Microb 80 (23): 7378-7387.

Teixeira E.W., L.G. Santos, A. Sattler, D. Message, M.L. Alves, et al. (2013) Nosema ceranae has been present in Brazil for more than three decades infecting Africanized honey bees. Journal of invertebrate pathology 114 (3): $250-254$.
Vásquez A., E. Forsgren, I. Fries, R.J. Paxton, E. Flaberg, et al. (2012) Symbionts as major modulators of insect health: lactic acid bacteria and honeybees. PloS one 7(3): e33188.

Zander E. (1909) Tierische parasiten als krankenheitserreger bei der biene. Münchener Bienenzeitung 31 : 196-204.

Publisher's note Springer Nature remains neutral with regard to jurisdictional claims in published maps and institutional affiliations. 